\title{
Heritability of dispersal rate and other life history traits in the Glanville fritillary butterfly
}

\author{
M Saastamoinen \\ Department of Biological and Environmental Sciences, University of Helsinki, Helsinki, Finland
}

\begin{abstract}
Knowing the variances and heritabilities $\left(h^{2}\right)$ of life history traits in populations living under natural conditions is necessary for a mechanistic understanding of respective evolutionary processes. I estimated heritabilities of several life history traits, including dispersal rate, body mass, age at first reproduction, egg mass, clutch size and lifetime reproductive success, in the Glanville fritillary butterfly (Melitaea cinxia) using parent-offspring regression. Experiments were conducted under field conditions in a large population cage $(32 \times 26 \mathrm{~m})$. Heritability estimates ranged from zero to almost one and several were significantly different from zero. Body size for both sexes, female age at
\end{abstract}

first reproduction and egg weight were all moderately to highly heritable, whereas heritabilities were low or nonexistent in clutch size and lifetime egg production. Heritability estimates for dispersal rate varied between the sexes, so that dispersal was heritable from mother to her female offspring only. This finding is consistent with previous results showing that the F1 female but not male offspring of females that naturally established new populations in the field are significantly more dispersive than butterflies in old populations.

Heredity (2008) 100, 39-46; doi:10.1038/sj.hdy.6801056; published online 5 September 2007

Keywords: heritability; dispersal; parent-offspring regression; life history; metapopulation

\section{Introduction}

Sufficient dispersal capacity is a fundamental prerequisite for a successful life history in most insects, as individuals typically need to fly to feed, mate and lay eggs. Dispersal may also help individuals cope with spatial and environmental heterogeneity (Clobert et al., 2001) as well as expand their ranges in changing environments (Hill et al., 1999; Travis and Dytham, 2002). The way that loss and fragmentation of natural habitats may affect the evolution of dispersal has received much attention in recent years (for example, Travis and Dytham, 1999; Van Dyck and Matthysen, 1999; Heino and Hanski, 2001; Hanski et al., 2004). These studies have shown that, depending on the species (life history) and the degree of the habitat loss, fragmentation may select for either increased or decreased dispersal rate (Heino and Hanski, 2001). Regardless of the direction of selection on dispersal due to fragmentation or other processes any evolutionary change assumes that dispersal rate is a heritable trait, which selection can act upon. Yet only few studies have tried to estimate heritability of dispersal rate directly, and none on butterflies, on which much research has been conducted on the likely consequences of habitat fragmentation (Thomas et al., 1998; Travis and Dytham, 1999; Thomas,

Correspondence: Ms M Saastamoinen, Department of Biological and Environmental Sciences, University of Helsinki, Viikinkaari 1, PO Box 65, Helsinki FIN-00014, Finland.

E-mail: marjo.saastamoinen@helsinki.fi

Received 27 March 2007; revised 6 August 2007; accepted 7 August 2007; published online 5 September 2007
2000) and climate change (Parmesan et al., 1999; Hill et al., 2003) on dispersal rate.

In the case of the Glanville fritillary butterfly (Melitaea cinxia) in the Aland Islands in Finland, the habitat is extremely fragmented, and thus movement between discrete habitat patches requires at least moderate dispersal capacity (Hanski, 1999). Increased flight capacity is beneficial especially in females, as it allows establishment of new populations and thereby for example helps avoiding inbreeding (Saccheri et al., 1998). On the other hand, there are also costs to high dispersal rate, in particular due to increased mortality and reduced time for reproduction (Hanski et al., 2006). Therefore, under certain conditions highly dispersive individuals may have reduced lifetime reproductive success and/or life expectancy (Hanski and Saccheri, 2006). Previous studies have shown that fitness benefits of being dispersive or sedentary in the Glanville fritillary are highly dependent upon the spatial configuration of populations and habitat patches (Hanski et al., 2006; Saastamoinen, 2007b).

The Glanville fritillary is regarded as a relatively sedentary species, as its lifetime dispersal distances are generally 1-2 km (Hanski et al., 2000) and the longest observed colonization distances are about $5 \mathrm{~km}$ (Hanski, 1999). However, there is much variation in the flight capacity among individuals (Saastamoinen, 2007b), and in particular Hanski et al. $(2002,2004)$ have shown that females originating from newly-established isolated populations are more dispersive in the field than those originating from old populations (based on a common garden mark-release-recapture experiment in the field). Comparable conclusions were drawn from a theoretical model and from physiological studies on the [ATP]/ 
[ADP] ratio in the flight muscles after constant forced flight (Hanski et al., 2004) and on the metabolic rate of females during active flight (Haag et al., 2005). Furthermore, and importantly for the present study, females originating from newly-established populations have higher early-life mobility than old population females when mobility was measured in a large outdoor population cage (Hanski et al., 2006; Saastamoinen, $2007 \mathrm{~b}$ ). These results have been explained by females establishing new populations, especially at isolated habitat patches, having greater dispersal propensity and/or dispersal capacity than the average female (Hanski et al., 2002). This assumes that variation in dispersal propensity and/or capacity is due to one or more heritable traits, but so far direct measurements of heritability of any relevant traits have been lacking.

An intriguing result in the Glanville fritillary studies described above is that in all cases the difference in dispersal rate or related traits between individuals from newly-established versus old populations is only present in females (Hanski et al., 2002, 2004, 2006). This may relate to the fact that even though both sexes disperse, dispersal has a different function in the two sexes. In females, flight is necessary for searching for nectar and oviposition sites within habitat patches as well as for dispersal to other patches, whereas in males mate searching is likely to be the key function of flight. Variation in dispersal capacity among males may be related to, for example, different mate searching strategies (perchers versus patrollers; Van Dyck and Matthysen, 1998) rather than to the ability to disperse from one habitat patch to another. Kuussaari et al. (1996) showed that in the Glanville fritillary males move more than females within habitat patches whereas females move more and longer distances than males between patches.

There are studies on insects that have investigated the heritability of dispersal capacity, either directly or by using surrogates such as wing length, but these studies have been mostly conducted in the laboratory (Dingle, 1991 and references therein). It is possible that estimating heritability under laboratory conditions may not yield representative results for natural populations. Only a few studies have attempted to estimate heritability of dispersal capacity under natural or semi-natural field conditions (Keil et al., 2001), most likely because of the difficulty of measuring dispersal rate in the field. Field studies also potentially suffer of increased phenotypic variation under variable natural environmental conditions (Roff, 2002).

The aim of the present study was to estimate heritability of dispersal rate in the Glanville fritillary under the relatively natural conditions in a large outdoor population cage $(32 \times 26 \mathrm{~m})$. As dispersal capacity is closely related to other reproductively important life history traits (Saastamoinen, 2007b), I also assessed the heritability of these other traits, including body mass, age at first reproduction, egg weight, clutch size and lifetime egg production.

\section{Materials and methods}

\section{Study species and populations}

In Finland, the Glanville fritillary butterfly ( $M$. cinxia) occurs in the Aland Islands only, where it has a classic metapopulation (Hanski, 1999). Of the roughly 4000 habitat patches in the entire study area, about 500 patches (small dry meadows) are occupied in any given year (Hanski, 1999). There is a high rate of population turnover, with around 100 (mostly very small) local populations going extinct in each year and roughly the same number of new populations being established (Nieminen et al., 2004). The Glanville fritillary has a univoltine life-cycle in the Alland Islands (Hanski, 1999; Nieminen et al., 2004), and the flight season lasts from early June to mid-July. Females eclose with the full complement of oocytes in their ovarioles and they typically mate soon after eclosion (Saastamoinen, 2007a). Females lay eggs in clutches of 130-160 eggs (Saastamoinen, 2007a), and they can lay up to 1200 eggs in their lifetime (Hanski et al., 2004; Saastamoinen, 2007b). Larval survival is positively correlated with larval group size (Kuussaari et al., 2004), and a number of female traits, including mobility, affect both clutch size and lifetime egg production (Saastamoinen, 2007a, b).

Parent generation: In spring 2005, around 400 5th instar larvae were collected from 60 local populations around the Aland Islands to include the full range of genetic variation across the metapopulation. The larvae were reared in the laboratory under constant environmental conditions (12:12 L/D, 25 and $20^{\circ} \mathrm{C}$, respectively) with fresh plant material available at all times. Individual pupae were weighed $24 \mathrm{~h}$ after pupation with a Scaltec SBC 33 electrobalance (accuracy $0.1 \mu \mathrm{g}$ ) to determine body mass. Adult butterflies (125 and 117 females and males, respectively) were released into the population cage (below) on the day of eclosion (some butterflies eclosed in the evening and were released in the following morning). Prior to their release into the cage butterflies were calmed down by keeping them a short while (5$10 \mathrm{~min})$ in $+5^{\circ} \mathrm{C}$, after which they were weighed and individually numbered underneath the hind wing by a permanent marker.

Offspring generation: Offspring of the females that reproduced in the population cage in 2005 were reared under the same conditions as their parents (12:12 L/D, 25 and $20^{\circ} \mathrm{C}$, respectively) in the laboratory until diapause. After reaching the diapause the larvae were kept in small containers with no more than 50 larvae per container and placed in a storeroom with temperature around $+3{ }^{\circ} \mathrm{C}$. In 2006, 200 offspring (106 females and 94 males) from 33 randomly selected females were reared to adulthood. An identical experiment to that conducted on the parents was conducted with the offspring generation. The mother of all individuals was known and for 143 individuals both the mother and the father were known, as the mating was directly observed in the experiment on the parent generation. Offspring of each female are assumed to be full siblings as females only rarely mate more than once (Saastamoinen, 2007b) and in the experiment only larvae from the first clutch of eggs laid by each female were used.

\section{Experimental set-up}

The experiments on individually marked butterflies were conducted in a large population cage $(32 \times 26 \times 3 \mathrm{~m})$ built upon a natural habitat (dry meadow) in the field (Hanski et al., 2006). The mesh that covers the cage prevents 
butterflies from escaping yet allows natural environmental conditions (rain, sun and wind) inside the cage. Potted host plants, Plantago lanceolata $(n=100)$ and Veronica spicata $(n=100)$, were provided for oviposition in the central part of the cage, which was relatively bare of vegetation compared with the edges of the cage. The edges had higher vegetation and greater numbers of flowering plants that provided nectar for butterflies. The cage was divided into $8 \times 8$ grid cells, which were surveyed systematically every second hour to gain information about the location and mobility (below) of butterflies. During these surveys mating pairs of butterflies were carefully recorded. Extra surveys were conducted during the warmer hours of the day to minimize the number of matings missed. Host plants were monitored continuously for ovipositing females.

The leaf on which the female laid her egg clutch was removed and the eggs were counted at the age of 3 days to determine the clutch size. The eggs and the larvae were reared in the laboratory under constant environmental conditions. Lifetime egg production was the sum of all clutches a female laid during the experiment. Egg clutches were gently separated from the leaf on which they were laid and weighed at the age of 5-6 days with a Scaltec SBC 33 electrobalance (accuracy $0.1 \mu \mathrm{g}$ ). The mean weight of individual eggs was obtained by dividing the weight of the clutch by the number of eggs. If there was any dirt in the egg clutch due to its position on the host plant (that is on a leaf that had been in contact with the ground) the clutch was omitted from the analysis.

As a measure of mobility in the cage I used the residual from the regression of the number of grid cells in which the butterfly had been observed against the number of observations during the surveys (Hanski et al., 2006). The mobility measure was calculated separately for three periods in the life of each butterfly, from 0 to 3 days, from 4 to 8 days and 9 days and more, as previous studies have shown that it is the early-life mobility (0-3 days) in the cage that varies between females from newly-established versus old populations (Hanski et al., 2006).

\section{Heritability estimates}

Heritability estimates for the different life history traits were obtained from the regression of offspring on parents. A mid-parent to mean offspring regression was done to estimate heritabilities for body mass and mobility. Single-parent to mean offspring regressions were done to find out the effects of mother and father separately on the heritability estimates. The phenotypic values were standardized for sex differences by subtracting the sex-specific mean value from each individual phenotypic value and dividing this by the sex-specific s.d. (Lynch and Walsh, 1998). If the first heritability estimate detected no significant resemblance between parents (mid-parent or mother and father alone) and their offspring, a second set of analyses was performed separately for male and female offspring. As the phenotypic variances of body weight and mobility varied between the sexes, adjustment factors based on s.d. were used (male s.d. $=x$, female s.d. $=y$, male/ female s.d. $=x / y$, female $/$ male s.d. $=y / x$, Falconer and Mackay, 1996).
Mother- and her female-offspring regressions were conducted to estimate heritabilities of reproductive traits: age at first reproduction, egg weight, clutch size and lifetime egg production. As the variances of traits need to be equivalent between the parent and offspring generations (Lynch and Walsh, 1998), heritability estimates were calculated for standardized phenotypic values. Both original (not corrected for body size) and sizecorrected (regressing egg weight against body mass) values for egg size were used, as egg weight may depend on body size. As the number of offspring per family varied all regressions were weighted as suggested by Roff (2002). The weighting did not have much effect on the results, as family sizes did not vary greatly.

Phenotypic correlations among life history traits that yielded positive heritability estimates from motherdaughter regressions were calculated with standardized pooled data on mothers and daughters. The respective estimates of genetic correlations and their standard errors were calculated by using covariances of each character as explained in Falconer and Mackay (1996).

\section{Data analysis}

All statistical analyses were performed with SAS (version 8.02; SAS Institute 1999). Linear models (GLM procedure in SAS) were used to test whether the resemblance between parents and offspring varied between the sexes.

\section{Results}

\section{Phenotypic values}

The phenotypic values of the life history traits for both the parent and offspring generations are summarized in Table 1. As the experiments were conducted under field conditions some variation between the generations inevitably occurs due to differences in environmental conditions between the years. In both males and females, the parent generation had somewhat heavier individuals than the offspring generation $\left(t_{(105)}=-4.53, P<0.001\right.$ and $t_{(133)}=-5.55, P<0.001$, respectively), and females in the parent generation started to reproduce later in their life than in the offspring generation $\left(t_{(104)}=-5.97, P<0.001\right)$. The average clutch size and lifetime egg production were also slightly different in the two generations $\left(t_{(104)}=-1.87\right.$, $P=0.071$ and $t_{(104)}=-2.28, P=0.025$, respectively)

Table 1 Average phenotypic values of life history traits in the parent and offspring generations

\begin{tabular}{lcc}
\hline Trait & $\begin{array}{c}\text { Parent } \\
\text { generation } \\
\text { mean }( \pm \text { s.d. })\end{array}$ & $\begin{array}{c}\text { Offspring } \\
\text { generation } \\
\text { mean }( \pm \text { s.d. })\end{array}$ \\
\hline Body weight $(\mathrm{mg})$ & & \\
$\quad$ Female & $227.4( \pm 21.1)$ & $199.6( \pm 24.1)$ \\
Male & $177.8( \pm 14.4)$ & $161.4( \pm 16.8)$ \\
Clutch size & $150.0( \pm 44.1)$ & $129.7( \pm 40.8)$ \\
Lifetime egg production & $548.6( \pm 280.1)$ & $410.1( \pm 260.7)$ \\
Age at first reproduction (days) & $5.7( \pm 2.31)$ & $2.7( \pm 1.34)$ \\
Egg weight (mg) & $0.083( \pm 0.007)$ & $0.089( \pm 0.008)$ \\
\hline
\end{tabular}


Table 2 Heritability estimates $\left(h^{2}\right)$ from regressions of mid-parent, male-parent only and female-parent only on mean offspring value for body mass and mobility (0-3 days old butterflies)

\begin{tabular}{|c|c|c|c|c|c|c|c|c|}
\hline \multirow{2}{*}{$\begin{array}{l}\text { Phenotypic } \\
\text { trait }\end{array}$} & \multicolumn{2}{|c|}{ Mid-parent } & \multicolumn{3}{|c|}{ Male parent } & \multicolumn{3}{|c|}{ Female parent } \\
\hline & Slope $=h^{2}$ & s.e. & Slope & s.e. & $h^{2}$ & Slope & s.e. & $h^{2}$ \\
\hline Bod & $0.361^{*}$ & 0.182 & 0.223 & 0.191 & 0.42 & $8^{* *}$ & 0.124 & 0.60 \\
\hline $\begin{array}{l}\text { Mobility } \\
\text { (0-3 days) }\end{array}$ & -0.0 & 0.104 & -0.0 & 0.081 & -0.09 & 0. & 0.081 & \\
\hline
\end{tabular}

Phenotypic values were standardized for the two sexes and regressions were corrected for unequal family sizes.

$* P<0.07$.

**P $<0.05$

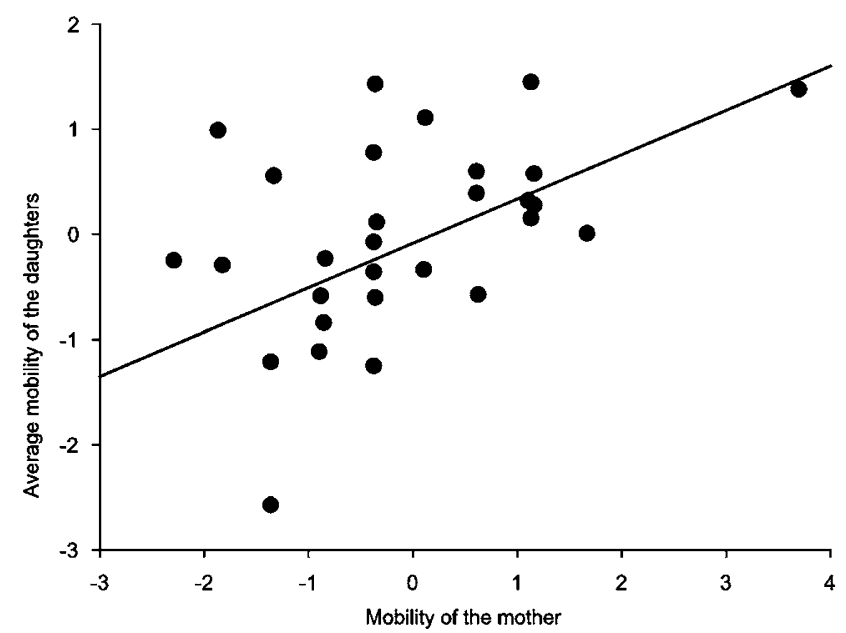

Figure 1 Mother-daughter regression for early-life mobility $(<4$ days) in 29 females and their daughters. For statistics see Table 3.

\section{Heritability estimates}

The heritability estimates for body mass and mobility from mid-parent-offspring and one-parent-offspring regressions are given in Table 2. Heavier parents produced heavier offspring but the resemblance was only weak (Table 2). The effect of mother's weight on offspring weight was much stronger than the effect of father's body weight (Table 2).

Heritability estimates for early-life mobility (0-3 days of age) from both mid-parent and one-parent regressions were close to zero (Table 2). However, when different sexes of the offspring were analyzed separately, it became evident that one combination differed significantly from the others; the more mobile females produced more mobile female offspring (Figure 1), and the estimate of heritability was significant and relatively high $\left(h^{2}=0.61\right.$; Table 3$)$. A nearly significant interaction term between mother's mobility and the sex of the offspring in a covariance analysis confirmed that the resemblance between the mother and her offspring was dependent on the sex of the offspring (ANCOVA, mother's mobility $\times$ sex, $\left.F_{1,152}=3.3, P=0.07\right)$. When mobility was calculated for the other two age classes, 4-8 days and more than 9 days, the heritability estimates were not significantly different from zero ( $P$-values between 0.24 and 0.83 for the different combinations).

The heritability estimate for female age at first reproduction was high $\left(h^{2}=0.78\right)$ and significant (Table 4 and Figure 2a). Egg mass not corrected for body mass
Table 3 Heritability estimates for mobility ( $0-3$ days of age) from mother to daughter, mother to son, father to daughter and father to son regressions

\begin{tabular}{lcccr}
\hline Type of regression & $\mathrm{F}$ & $\mathrm{P}$ & $\mathrm{r}^{2}$ & \multicolumn{1}{c}{$h^{2}$ (s.e.) } \\
\hline Mother-daughter $^{2}$ & 6.93 & 0.015 & 0.240 & $0.61(0.24)$ \\
Mother-son $^{\mathrm{a}}$ & 2.15 & 0.157 & 0.093 & $-0.39(0.28)$ \\
Father-daughter $^{\mathrm{a}}$ & 1.22 & 0.288 & 0.080 & $-0.29(0.26)$ \\
Father-son & 0.88 & 0.362 & 0.052 & $0.25(0.26)$ \\
\hline
\end{tabular}

Regressions are corrected for unequal family sizes.

${ }^{a}$ Heritability estimates are corrected for phenotypic variance between the sexes.

Table 4 Heritability estimates $\left(h^{2}\right)$ from mother to daughter regressions for age at first reproduction, egg weight, clutch size and lifetime egg production

\begin{tabular}{lcccr}
\hline Phenotypic trait & $\mathrm{F}$ & $\mathrm{P}$ & $\mathrm{r}^{2}$ & \multicolumn{1}{c}{$h^{2}$ (s.e.) } \\
\hline Age at first reproduction & 5.98 & 0.030 & 0.315 & $0.78(0.32)$ \\
Egg weight (uncorrected) & 3.97 & 0.062 & 0.181 & $0.68(0.34)$ \\
Body mass-corrected egg weight & 7.27 & 0.015 & 0.288 & $0.97(0.36)$ \\
Clutch size & 1.62 & 0.219 & 0.079 & $-0.38(0.32)$ \\
Lifetime egg production & 0.07 & 0.799 & 0.004 & $0.09(0.36)$ \\
\hline
\end{tabular}

Estimates are calculated for standardized phenotypic values, and regressions are corrected for unequal family sizes.

was almost significantly heritable, and for body masscorrected egg mass the heritability estimate was very high and significant (Table 4 and Figure 2b). Of the female reproductive traits, heritability estimates for clutch size and lifetime egg production were very low and not significant due to relatively large s.e. (Table 4 and Figures 2c and d, respectively).

\section{Genetic and phenotypic correlations among the life history traits}

Table 5 shows both genetic (values below diagonal) and phenotypic (values above diagonal) correlations among the traits that showed positive heritabilities between mother and her female offspring. Calculating genetic correlations with relatively small sample sizes from natural populations involves problems and therefore s.e. are often high (Falconer and Mackay, 1996). Positive genetic correlation, with relatively small s.e., was detected only between body mass and egg weight. There was also a significant phenotypic correlation in the pooled data $(n=103)$ for these traits. There is a negative genetic correlation between body mass and early-life mobility, but the phenotypic correlation was not significant in this case (Table 5). All other correlations were non-significant (Table 5).

\section{Discussion}

Four of the six life history traits included in this study were significantly heritable, with values of $h^{2}$ ranging from 0.29 to 0.97 . These values can be considered to be moderate to high as heritability estimates for life history traits generally average around 0.25 (Roff, 2002; Roff and Fairbairn, 2007). Heritabilities of clutch size and lifetime egg production did not differ from zero, suggesting that 

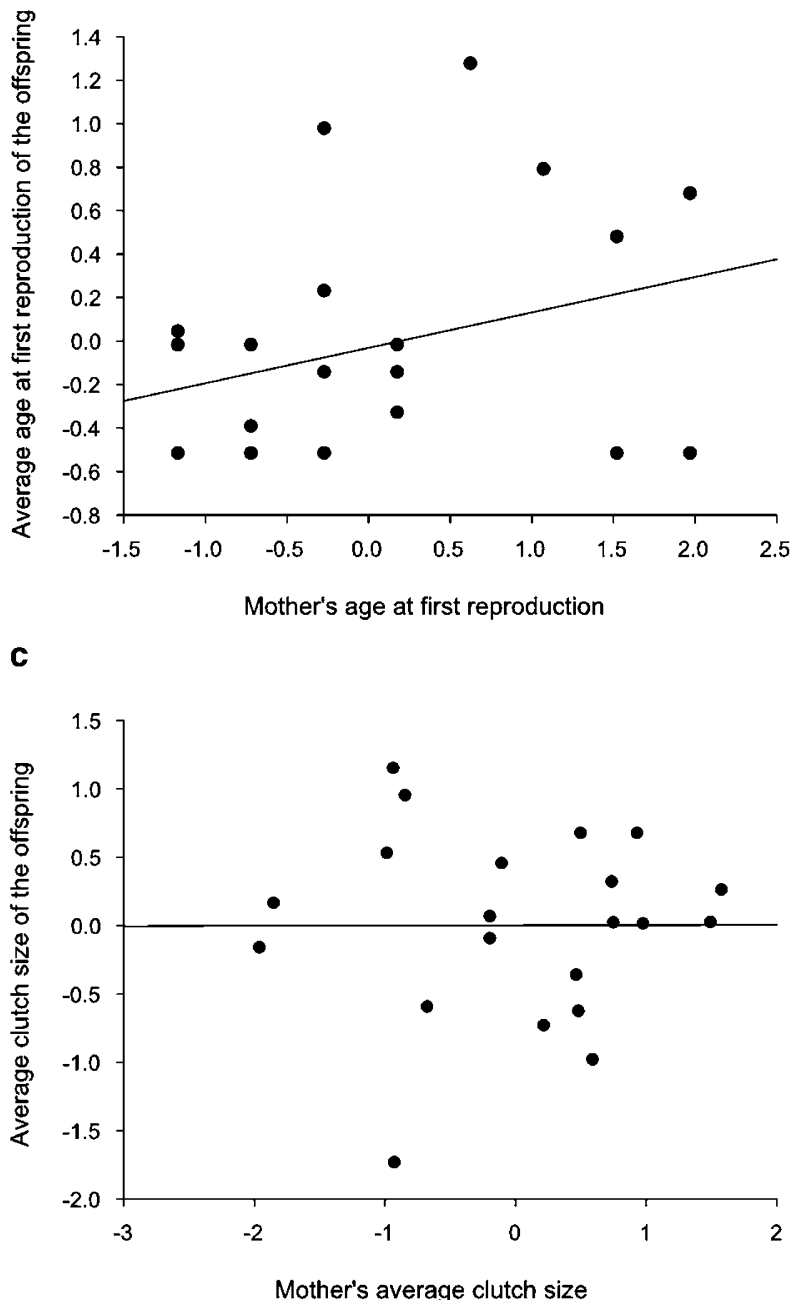

b

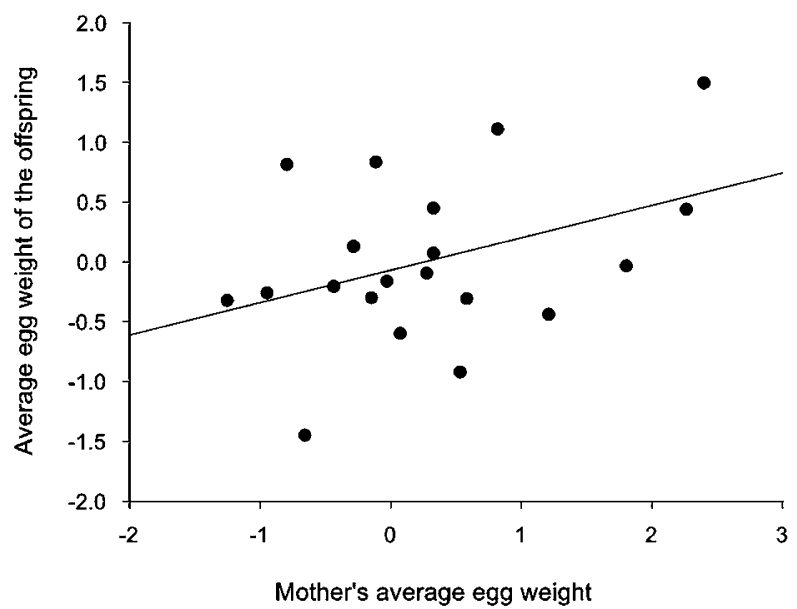

d

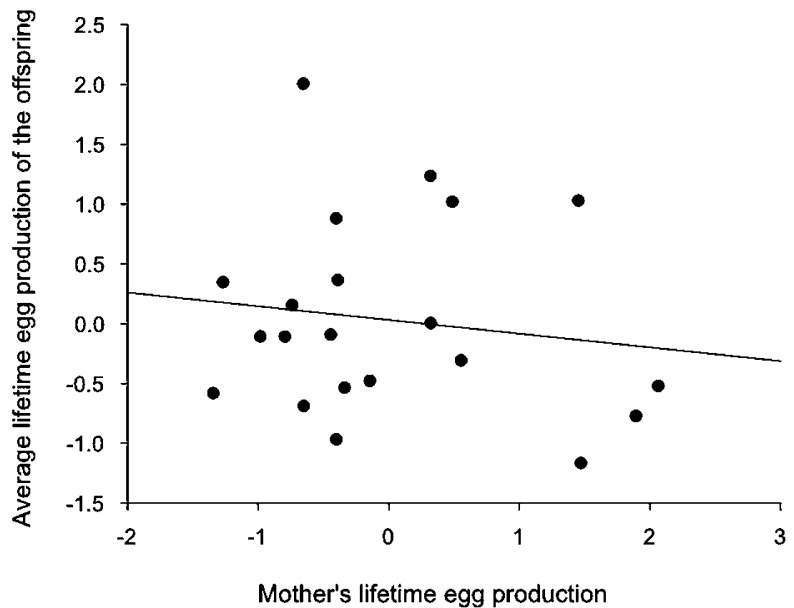

Figure 2 Mother-daughter regressions for age at first reproduction (a), egg size (b), clutch size (c) and lifetime egg production (d). Age at first reproduction and egg size were significantly heritable, whereas no significant heritability was detected for clutch size or lifetime egg production. All phenotypic values were standardized for equal variances. For statistics see Table 4.

Table 5 Phenotypic correlations (above diagonal) and genetic correlations (below diagonal) between life history traits

\begin{tabular}{lcccc}
\hline & Body mass & Mobility & Age at first reproduction & Egg weight \\
\hline Body mass & - & -0.104 & -0.099 & $-0.237^{*}$ \\
Mobility & $-0.516(0.15)^{* *}$ & - & -0.042 \\
Age at first reproduction & $-0.224(0.20)$ & $-0.199(0.28)$ & -152 & -0.032 \\
Egg weight & $0.599(0.15)^{* *}$ & $0.175(0.30)$ & - \\
\hline
\end{tabular}

Genetic correlations are followed by s.e. within parentheses.

$* P<0.02$.

$* * P<0.01$.

the values of these traits may be mainly determined by environmental conditions. This result is consistent with the expectation that selection reduces additive genetic variation of traits closely linked with fitness (Fisher, 1930; Mousseau and Roff, 1987).

There has been much research recently on dispersal in the Glanville fritillary (Hanski et al., 2002; Haag et al., 2005; Hanski and Saccheri, 2006). Systematic differences between females originating from newly-established versus old populations in traits directly or indirectly related to dispersal are most naturally explained by non- random trait values of the founders of new populations, which are expected to be more dispersive than the average individual, and by inheritance of these traits by the offspring of the founding females. The present results demonstrate that mobility was highly heritable from mother to daughter $\left(h^{2}=0.61\right)$ but not from mother to son or from father to daughter or son. It is particularly noteworthy that in the natural populations, too, only females but not males in newly-established populations are especially dispersive (Hanski et al., 2004, 2006). Another consistent result relates to the mobility of 
different-aged individuals in the population cage. A previous study found that only young (0-3 days old) females exhibited the difference in mobility between newly-established and old populations (Hanski et al., 2006). Here the heritability estimate of mobility was different from zero only in the case of early-life mobility.

A possible explanation of mobility being heritable only from mother to her female offspring is that the trait is sex-linked. In butterflies, females are the heterogametic sex (XY, also referred to as WZ) and males are homogametic ( $\mathrm{XX}$ or $\mathrm{ZZ})$. The resemblance between mother and her female offspring is therefore consistent with mobility being controlled by a locus or loci on the $Y$ chromosome that is either absent from $X$ or is present but suppressed (Nijhout, 1991). An alternative and more likely explanation is that mobility is dissimilarly expressed in the two sexes. The function of early-life mobility in males is likely to be primarily mate searching as most females only mate once and do so soon after eclosion (Saastamoinen, 2007b). The measure of mobility used in the cage may not distinguish well differences in male movement behaviour, namely differences in mate searching strategies (Van Dyck and Matthysen, 1998). In females, on the other hand, flight is necessary for searching for nectar and oviposition sites within habitat patches as well as for dispersal to other patches, and any differences in mobility among individuals may be easier to distinguish than in males. Possible maternal effects on mobility are discussed below.

Heritability estimates for dispersal-related traits in insects have been measured either directly or indirectly in numerous studies (Parker and Gatehouse, 1985; Dingle, 1991; Gu and Danthanarayana, 1992; Schumacher et al., 1997; Keil et al., 2001; Roff and Fairbairn, 2001 and references therein) and the estimates are often high, $h^{2} \sim 0.5$ or more (Dingle, 1991; Roff and Fairbairn, 2001 and references therein). Most of these studies were conducted in the laboratory under constant environmental conditions and hence the applicability of the results to natural populations is questionable. The study of Keil et al. (2001) is an important exception. They showed that estimated heritabilities of mobility in the codling moth (Cydia pomonella) were 0.43 in males and 0.29 in females based on two directional selection experiments, and they further demonstrated that dispersive and sedentary morphs performed accordingly when their dispersal rate was measured in the field (Keil et al., 2001).

High heritability of and apparently large genetic variation in the dispersal capacity of the Glanville fritillary raise questions about the factors responsible for the maintenance of such variation. Previous studies have indicated that the more mobile females acquire mates sooner and also initiate oviposition earlier than the more sedentary females (Saastamoinen, 2007b). Age at first reproduction is an important life history trait in many species (Roff, 2002). On the other hand, the reproductive significance of mobility in the Glanville fritillary depends on the age of the respective local population. In newly-established populations, where females are more mobile (Hanski et al., 2002), mobility is positively correlated with lifetime reproductive success, whereas in old populations the opposite is true, and more mobile females have reduced lifetime egg production compared with sedentary females (Saastamoinen, 2007b). It is therefore possible that genetic variation in mobility in the Glanville fritillary is maintained by spatial variation in the selection pressures between different population types in the metapopulation as a whole. In general, between-patch selection (colonization) favours dispersive individuals while withinpatch selection favours more sedentary individuals, which would allow metapopulation-level coexistence of phenotypes with different dispersal rates (Olivieri et al., 1995).

Genetic variability of phenotypic traits that are selected for can be maintained if such traits are condition-dependent or if there exist trade-offs with other reproductively important traits (Roff, 1992, 2002). A negative genetic correlation between early-life mobility and body mass in the present study suggests a tradeoff between these two traits. The correlation was insignificant when measured at the phenotypic level, which could be due to the 'silver spoon effect', whereby negative genetic correlation is masked by both traits being affected by the same favourable or unfavourable environmental factors (Grafen, 1988). Alternatively, lack of phenotypic correlation may result from variation in allocation and resource acquisition patterns between dispersive- and non-dispersive individuals. In any case, genetic correlation between mobility and body size could act as a constraint to the independent evolution of these traits in the Glanville fritillary.

Body mass (pupal weight) was moderately heritable $\left(h^{2}=0.36\right)$, but a closer analysis indicated that the offspring resemble more closely their mother than their father and hence this correlation could be due to maternal effects (see below). Studies on other Lepidoptera have shown moderate to high heritability of pupal weight (for example, Bicyclus anynana; BJ Zwaan et al., unpublished in Fischer et al., 2004). Body mass in butterflies often correlates with egg and clutch sizes. In the present study there was moderately high positive genetic as well as phenotypic correlation between body mass and egg weight. Stabilizing selection may maintain genetic variation (Roff, 2002) in body mass as selection may favour smaller individuals due to their shorter development time and earlier emergence (Wiklund et al., 1991). In the Glanville fritillary, the relationship between body size and development time has been inconsistent in different years (Saastamoinen, 2007a).

Mothers that laid heavy eggs produced offspring that also laid heavy eggs, and the heritability estimate was even higher when egg weight was corrected for female body mass. As maternal effects are likely to be mediated through maternal condition, which in turn is likely to be reflected in body mass, this result implies that maternal effects alone do not explain the resemblance between mothers and daughters. In the butterfly B. anynana, heritability of egg size was found to range from low to moderate $\left(h^{2} \sim 0.4\right)$ based on parent-offspring regression (Fischer et al., 2004). There is likely to be a fitness advantage of laying heavier eggs in the Glanville fritillary, as egg hatching rate is positively correlated with egg weight (Saastamoinen, 2007b).

Age at first reproduction is often considered to be a key life history trait, which correlates with female lifetime fecundity (for example Roff and Fairbairn, 1991), partly because early maturing individuals have higher probability of surviving to maturity (Stearns, 
1992). In the Glanville fritillary, females eclose with the full complement of oocytes in their ovarioles, and hence the age at first oviposition reflects either egg maturation rate, the rate at which the female acquired a mate, or both. Delayed age at first reproduction can in some cases be compensated for by further growth or increased lifespan, and may hence have fitness benefits (Roff et al., 2006). As body size is determined at eclosion in butterflies the former hypothesis is not plausible. Whether individuals that delay their age at first oviposition have longer lifespan is not known for the Glanville fritillary, but this is likely to depend on the environmental conditions and thus vary between years. In the present study age at first oviposition was highly heritable from mothers to daughters. As discussed above, maintenance of variation in this trait is likely to be due to environmental plasticity, as the benefits of reproducing at an early age are surely condition dependent and vary between the years.

A potential problem in estimating heritabilities with parent-offspring regression in populations under field conditions is that assessing the amount of maternal effects on the additive genetic variance is not possible and that the common environment affecting the traits of both the mothers and the offspring may bias heritability estimates (Falconer and Mackay, 1996). The latter is however not a problem in the present study as different generations were faced with different natural environmental conditions. On the other hand, the environmental conditions the mothers faced during their development may affect their offspring, which might cause overestimation of the heritabilities of some traits. Body mass and egg mass are two traits that may well be affected by maternal effects, but it is less obvious how maternal effects could affect the heritability estimates of mobility, especially as mobility was significantly heritable only from mothers to daughters.

Recent studies on birds have shown that maternal effects can be strongly sex-specific and apparently play an important role in the evolution of sexual dimorphism (Badyaev, 2005; Badyaev et al., 2005, 2006). Sex-specific maternal effects can arise by for example, mothers allocating variable amounts of nutrients or hormones to sons and daughters in relation to their own condition (Badyaev et al., 2005, 2006). Whether in the present study the estimation of heritability of mobility between mother and her female offspring is inflated by maternal effects only expressed in females remains an unanswered but intriguing possibility.

\section{Conclusion}

These results support the previous inferences from observational data that dispersal rate is heritable in the Glanville fritillary, though only from the mother to her female offspring (Hanski et al., 2002, 2004, 2006). The most likely explanation for the maintenance of variation in dispersal rate is spatially varying selection pressures in the metapopulation, for which there is also direct empirical evidence (Hanski and Saccheri, 2006; Saastamoinen, 2007b). The present results are among the first to demonstrate significant heritability for dispersal in an insect population under field conditions.

\section{Acknowledgements}

I thank J Alho, J Brommer, P Gienapp and O Ovaskainen for help in the analysis, and M Björklund, J Brommer, I Hanski and two anonymous referees for comments on the manuscript. Thanks to many people who took part in the population cage experiments and helped rearing the larvae in 2005 and 2006. Regional Council of the Aland Islands is acknowledged for permitting the use of their land for the experiments. Financial support for the research was provided by the Academy of Finland (Finnish Center of Excellence Programme in 2003-2005 and 2006-2008, Grant nos. 20286 and 213457).

\section{References}

Badyaev AV (2005). Maternal inheritance and rapid evolution of sexual size dimorphism: passive effects or active strategies? Am Nat 166: S17-S30.

Badyaev AV, Acevedo Seaman D, Navara KJ, Hill GE, Mendonca MT (2006). Evolution of sex-biased maternal effects in birds: III. Adjustment of ovulation order can enable sex-specific allocation of hormones, carotenoids, and vitamins. J Evol Biol 19: 1044-1057.

Badyaev AV, Schwabl H, Young RL, Duckworth RA, Navara KJ Parlow AF (2005). Adaptive sex differences in growth of preovulation oocytes in a passerine bird. Proc R Soc Lond B 272: 2165-2172.

Clobert J, Danchin E, Dhondt AA, Nichols JD (2001). Dispersal. Oxford University Press: New York.

Dingle H (1991). Evolutionary genetics of animal migration. Am Zool 31: 253-264.

Falconer DS, Mackay TFC (1996). Quantitative Genetics. Longman Group Ltd: Harlow, UK.

Fischer K, Zwaan BJ, Brakefield PM (2004). Genetic and environmental sources of egg size variation in the butterfly Bicyclus anynana. Heredity 92: 163-169.

Fisher RA (1930). The Genetical Theory of Natural Selection. Clarendon press: Oxford.

Grafen A (1988). On the uses of data on lifetime reproductive success. In: Clutton-Brock TH (ed). Reproductive Success. Chicago Press: Chicago, IL, pp 454-463.

Gu H, Danthanarayana W (1992). Quantitative genetic analysis of dispersal in Epiphyas postvittana. I. Genetic variation in flight capacity. Heredity 68: 53-60.

Haag CR, Saastamoinen M, Marden JH, Hanski I (2005). A candidate locus for variation in dispersal rate in a butterfly metapopulation. Proc $R$ Soc Lond B 272: 2449-2456.

Hanski I (1999). Metapopulation Ecology. Oxford University Press Inc.: New York.

Hanski I, Breuker CJ, Schöps K, Setchfield R, Nieminen M (2002). Population history and life history influence the migration rate of female Glanville fritillary butterflies. Oikos 98: 87-97.

Hanski I, Erälahti C, Kankare M, Ovaskainen O, Siren H (2004). Variation in migration propensity among individuals maintained by the landscape structure. Ecol Lett 7: 958-966.

Hanski I, Saastamoinen M, Ovaskainen O (2006). Dispersalrelated life history trade-offs in a butterfly metapopulation. J Anim Ecol 75: 91-100.

Hanski I, Saccheri I (2006). Molecular-level variation affects population growth in a butterfly metapopulation. PLoS Biology 4: 0719-0726.

Hanski I, Alho J, Moilanen A (2000). Estimating the parameters of survival and migration of individuals in metapopulations. Ecology 81: 239-251.

Heino M, Hanski I (2001). Evolution of migration rate in spatially realistic metapopulation model. Am Nat 157: 495-511. 
Hill JK, Thomas CD, Huntley B (2003). Modeling present and potential future ranges of European butterflies using climate response surfaces. In: Boggs C, Watt WB, Ehrlich PR (eds). Butterflies. Ecology and Evolution of Taking Flight. The University of Chicago Press: Chicago, pp 149-167.

Hill JK, Thomas CD, Lewis OT (1999). Flight morphology in fragmented populations of a rare British butterfly, Hesperia comma. Biol Conserv 87: 277-283.

Institute S (1999). SAS/STAT Software User's Guide, Release 8.00. SAS Institute Inc: Cary.

Keil S, Gu HN, Dorn S (2001). Response of Cydia pomonella to selection on mobility: laboratory evaluation and field verification. Ecol Entomol 26: 495-501.

Kuussaari M, Nieminen M, Hanski I (1996). An experimental study of migration in the Glanville fritillary butterfly Melitaea cinxia. J Anim Ecol 65: 791-801.

Kuussaari M, van Nouhuys S, Hellmann J, Singer M (2004). Larval biology. In: Ehrlich PR, Hanski I (eds). On The Wings of Checkerspots: A Model System for Population Biology. Oxford University Press: Oxford, pp 138-160.

Lynch M, Walsh B (1998). Genetics and Analysis of Quantitative Traits. Sinauer Associates Inc.: Sunderland, Massachusetts, USA.

Mousseau TA, Roff DA (1987). Natural selection and the heritability of fitness components. Heredity 59: 181-197.

Nieminen M, Siljander M, Hanski I (2004). Structure and dynamics of Melitaea cinxia metapopulations. In: Ehrlich PR Hanski I (eds). On the Wings of Checkerspot: A Model System for Population Biology. Oxford University Press: Oxford, pp 63-91.

Nijhout HF (1991). The Developmental Physiology of Colour Patterns in Lepidoptera. Smithsonian Institution Press: Washington, DC.

Olivieri I, Michalakis Y, Gouyon P-H (1995). Metapopulation genetics and the evolution of dispersal. Am Nat 146: 202-228.

Parker WE, Gatehouse AG (1985). Genetic factors controlling flight performance and migration in the African armyworm moth, Spodoptera exempta (Walker) (Lepidoptera: Noctuidae). Bull Entomol Res 75: 49-63.

Parmesan C, Ryrholm N, Stefanescu C, Hill JK, Thomas CD, Descimon $\mathrm{H}$ et al. (1999). Poleward shifts in geographical ranges of butterfly species associated with regional warming. Nature 399: 579-583.

Roff DA (1992). The Evolution of Life Histories. Theory and Analysis. Chapman \& Hall: New York.

Roff DA (2002). Life History Evolution. Sinauer Associates: Sunderland, MA.
Roff DA, Fairbairn DJ (1991). Wing dimorphism and the evolution of migratory polymorphism among the Insecta. Am Zool 31: 343-351.

Roff DA, Fairbairn DJ (2001). The genetic basis of dispersal and migration and its consequences for the evolution of correlated traits. Dispersal. Oxford University Press: New York, pp 191-202.

Roff DA, Fairbairn DJ (2007). The Evolution and Genetics of Migration in Insects. Bioscience 57: 155-164.

Roff DA, Heibo E, Vollestad LA (2006). The importance of growth and mortality costs in the evolution of the optimal life history. J Evol Biol 19: 1920-1930.

Saastamoinen M (2007a). Life-history, genotypic, and environmental correlates of clutch size in the Glanville fritillary butterfly. Ecol Entomol 32: 235-242.

Saastamoinen M (2007b). Mobility and lifetime fecundity in new versus old populations of the Glanville fritillary butterfly. Oecologia 153: 569-578.

Saccheri I, Kuussaari M, Kankare M, Vikman P, Fortelius W, Hanski I (1998). Inbreeding and extinction in a butterfly metapopulation. Nature 392: 491-494.

Schumacher P, Weber DC, Hagger C, Dorn S (1997). Heritability of flight distance for Cydia pomonella. Entomol Exp et Appl 85: 169-175.

Stearns SC (1992). The Evolution of Life Histories. Oxford University press: New York.

Thomas CD (2000). Dispersal and extinction in fragmented landscapes. Proc R Soc Lond B 267: 139-145.

Thomas CD, Hill JK, Lewis OT (1998). Evolutionary consequences of habitat fragmentation in a localized butterfly. I Anim Ecol 67: 485-497.

Travis JMJ, Dytham C (1999). Habitat persistence, habitat availability and the evolution of dispersal. Proc $R$ Soc Lond B 266: 723-728.

Travis JMJ, Dytham C (2002). Dispersal evolution during invasions. Evol Ecol Res 4: 1119-1129.

Van Dyck H, Matthysen E (1998). Thermoregulatory differences between phenotypes in the speckled wood butterfly: hot perchers and cold patrollers? Oecologia 114: 326-334.

Van Dyck H, Matthysen E (1999). Habitat fragmentation and insect flight: a changing 'design' in a changing landscape? TREE 14: 172-174.

Wiklund C, Nylin S, Forsberg J (1991). Sex-related variation in growth-rate as a result of selection for large size and protandry in a bivoltine butterfly, Pieris napi. Oikos 60: 241-250. 